\title{
Pelvic instability and trunk and hip muscle recruitment patterns in patients with total hip arthroplasty.
}

\section{$\operatorname{AUTHOR}(S)$ :}

Tateuchi, Hiroshige; Tsukagoshi, Rui; Fukumoto, Yoshihiro; Akiyama, Haruhiko; So, Kazutaka; Kuroda, Yutaka; Ichihashi, Noriaki

\section{CITATION:}

Tateuchi, Hiroshige ... [et al]. Pelvic instability and trunk and hip muscle recruitment patterns in patients with total hip arthroplasty.. Journal of electromyography and kinesiology : official journal of the International Society of Electrophysiological Kinesiology 2013, 23(1): 151-158

\section{ISSUE DATE:}

2013-02

URL:

http://hdl.handle.net/2433/169791

\section{RIGHT:}

(c) 2012 Elsevier Ltd.; この論文は出版社版でありません。引用の際には 出版社版をご確認ご利用ください。; This is not the published version. Please cite only the published version. 
Pelvic instability and trunk and hip muscle recruitment patterns in patients with total hip arthroplasty

Hiroshige Tateuchi ${ }^{\mathrm{a}, *}$, Rui Tsukagoshi ${ }^{\mathrm{a}}$, Yoshihiro Fukumoto ${ }^{\mathrm{b}}$, Haruhiko Akiyama ${ }^{\mathrm{c}}$, Kazutaka So $^{\mathrm{c}}$,

$5 \quad$ Yutaka Kuroda $^{\mathrm{c}}$, Noriaki Ichihashi ${ }^{\mathrm{a}}$

${ }^{a}$ Human Health Sciences, Graduate School of Medicine, Kyoto University, Kyoto, Japan

${ }^{b}$ Faculty of Rehabilitation, Kobe Gakuin University, Kobe, Japan

${ }^{c}$ Department of Orthopaedic Surgery, Kyoto University, Kyoto, Japan

10

Key words:

Total hip arthroplasty

Pelvic motion

15 Electromyography

Multifidus

* Corresponding author:

Hiroshige Tateuchi, PhD

Assistant Professor, Human Health Sciences, Graduate School of Medicine, Kyoto University

2553 Kawara-cho, Shogoin, Sakyo-ku, Kyoto 606-8507, Japan

Tel: +81-75-751-3964

Fax: +81-75-751-3909

E-mail: tateuchi@hs.med.kyoto-u.ac.jp 


\begin{abstract}
Hip and lumbar spine disorders often coexist in patients with total hip arthroplasty (THA). The current study aimed to reveal pelvic motion pathology and altered trunk and hip muscle recruitment patterns relating to pelvic motion in patients with THA. Twenty-one women who underwent THA and 12 age-matched healthy women were recruited. Pelvic kinematics and muscle recruitment patterns (i.e., amplitude, activity balance, and onset timing) of the gluteus maximus, semitendinosus, multifidus, and erector spinae were collected during prone hip extension. Compared with healthy subjects, the patients showed increased pelvic motion, especially ventral rotation, decreased multifidus muscle activity relative to the hip extensors, and delayed onset of multifidus activity, despite reaction times and speeds of leg motion not being significantly different between the groups. Furthermore, while contributing factors associated with ventral pelvic rotation were not found, delayed onset of multifidus activity was detected as a factor related to the increased anterior tilt of the pelvis $(r=0.47, p<0.05)$ in patients with THA. These results suggest that patients with THA have dysfunction of the stabilizer muscles of the lumbopelvic region along with increased pelvic motion.
\end{abstract}

\title{
1. Introduction
}

Hip and spine disorders commonly coexist in patients with hip osteoarthritis (McNamara et al., 1993; Stupar et al., 2010), and low back pain is reported by up to $49.4 \%$ of these patients (Parvizi et al., 2010). This pathological condition has been described as the hip-spine syndrome (Offierski and MacNab, 1983); restoration of hip biomechanics by total hip arthroplasty (THA) often resolves the related low back pain or lumbar spine disorders (Ben-Galim et al., 2007; Parvizi et al., 2010). However, Parvizi et al. (2010) showed that lumbar pain persists in 33.5\% of patients, even after THA. Interestingly, those patients with low back pain included patients who did not have preexisting spinal diseases, suggesting that patients undergoing THA with no apparent spinal disease are at risk for lumbopelvic disorders. Importantly, THA patients with lumbar spine disorders experience less improvement in hip pain and functional scores from the postoperative therapy, and cost the healthcare system about 1.4 times as much, compared to patients with THA alone (Prather et al., 2012).

Patients with limited ranges of hip motion and those having had THA tend to compensate by altering their lumbopelvic motion (Miki et al., 2004), which may result in the establishment of movement patterns associated with increased lumbopelvic motion. Hu et al. (2010) showed that adjunctive lumbopelvic stability using a pelvic belt results in less strain on hip muscles during leg 
raise compared with the motion without a pelvic belt, suggesting that lumbopelvic stability facilitates efficient use of hip muscles in addition to preventing destabilization of the lumbopelvis. Therefore, detailed evaluation and appropriate treatment for pathokinesiology of the hip and lumbopelvic region is a key consideration for patients following THA, regardless of the presence of low back pain.

Lumbopelvic instability has been assessed from 2 standpoints: mechanical and functional instability (Beazell et al., 2010; Panjabi, 2003). Mechanical instability is considered to be related to an increase in end range of motion assessed by imaging findings, including X-rays and magnetic resonance imaging, while functional instability appears to be related to a loss of segmental stiffness and mid-range segmental neuromuscular control during motion (Beazell et al., 2010). Functional instability has been evaluated by observing altered lumbopelvic motion during trunk and lower limb movements. In this study, we defined lumbopelvic instability (functional instability) as excessive motion and unsustainability of optimal alignment in the lumbopelvic region, compared with the motion and lumbopelvic alignment shown by healthy individuals, during lower limb movement.

Successful treatment of lumbopelvic instability is difficult, in part, because the pathology of the muscle recruitment patterns (i.e., amplitude, activity balance, and onset timing) and the relationship between altered muscle activity and the pelvic motion are not completely understood. Thus, the purposes of this study were (1) to reveal changes in pelvic motion and trunk and hip muscle activity in patients with THA and (2) to determine the relationship between pelvic motion and muscle recruitment patterns within patients with THA. The results of this study offer insight into treatment for lumbopelvic instability in patients with THA

\section{Methods}

\subsection{Participants}

Twenty-one women, who underwent THA at least 6 months prior to the study, were recruited. Their mean age was $62.5 \pm 6.6$ (mean \pm SD) years; with a mean body weight of $50.1 \pm 6.5 \mathrm{~kg}$; and an average height of $152.7 \pm 4.4 \mathrm{~cm}$. Since pain affects feedforward muscle activity and movement strategies (Dubois et al., 2011; Hodges et al., 2003), patients without hip pain were recruited. All patients had undergone THA due to painful hip osteoarthritis, and surgery had occurred, on average, $32.5 \pm 12.1$ months prior to the study. The average Harris hip score of the patients was $85.8 \pm 11.5$ (100 point muximum). Patients were excluded from the study if they had musculoskeletal conditions other than THA or if they had been diagnosed with neurological disorders. Twelve additional women, matched for age $(63.3 \pm 5.1$ years $)$, weight $(50.4 \pm 5.5 \mathrm{~kg})$, and height $(152.3 \pm 5.0 \mathrm{~cm})$, were also recruited; these control individuals were free from orthopedic and neurologic abnormalities. Participants provided informed consent, and the Institutional Ethics Committee approved the study.

\subsection{Experimental procedure}


Prone hip extension, commonly used as a self-perturbation task to test the lumbopelvic stability (Janda, 1996; Sahrmann, 2002), was used in this study. The participants were asked to lie on a table in the prone position with the right hip hanging over the edge of the table, which was tilted down to $30^{\circ}$ (Fig. 1). Each participant was instructed to perform active hip extension from $30^{\circ}$ to $0^{\circ}$ of hip flexion while keeping the knee extended; fixation devices were not applied to the pelvis or trunk. Movement during each trial started after a verbal ready signal followed by a random visual cue using 2 (right and left) light-emitting diodes (LED) set in front of the participants. The illumination of one of the LEDs indicated which leg the subject should raise. The participants were instructed to perform the task as rapidly as possible in response to the visual cue. Several practices were allowed, prior to testing, to familiarize the participants with the required movements. For the patients with THA, 5 tasks with the affected leg were included in the analysis; for the controls, 5 tasks with the nondominant leg (i.e., the leg opposite to the one the subjects would use for kicking a ball) were included. To avoid comparison with superior members, we adopted the nondominant leg in healthy individuals as control.

\subsection{Kinematic measurements}

Pelvic kinematics data were recorded using a Vicon Nexus (Vicon Motion System Ltd., Oxford, England) with 6 cameras operating at a sampling frequency of $200 \mathrm{~Hz}$. The subjects were clothed in close-fitting shorts and T-shirts, with 7 light-reflecting markers attached to anatomical landmarks on the bilateral posterior superior iliac spine, top of the iliac crest, right greater trochanter, lateral epicondyle of the femur, and lateral malleolus (Fig. 1). All data were low-pass filtered using a Woltring filter with a cut-off frequency of $6 \mathrm{~Hz}$.

Three-dimensional (3D) angular displacements (tilt, oblique, and rotation) of the pelvis were calculated over time (Fig. 1). To standardize the analytical range among participants, 3D angles of the pelvis were calculated as the changes from the angle in the initial prone position to the angle at which the knee marker reached $75 \%$ of the height of the initial position of the greater trochanter marker.

In addition to the amount of the pelvic angle change during movement, onset timing of the pelvic motion was determined. The onset of the pelvic motion was defined as the time at which the angular velocity of the anterior pelvic tilt exceeded $5 \%$ of the maximal angular velocity. The initiation of the leg motion was defined as the point at which the upward velocity of the marker on the lateral femoral epicondyle exceeded 5\% of the maximal velocity (Sholtes et al., 2009) (Fig. 2). We used a method with relative thresholds to determine the onset timing of the motion, taking into consideration that the range and velocity of the motion would differ among individuals. The termination of the leg motion was defined as the point at which the knee marker reached $75 \%$ of the height of the initial position of the greater trochanter marker. The onset time of the pelvic motion was normalized to the limb movement time by dividing the start-time difference by the time required to complete the leg movement (Sholtes et al., 2009) (Fig. 2). 


\subsection{Electromyography recording and data analysis}

The skin was carefully cleaned with ethanol before electrode placement. Bipolar surface electromyography (EMG) electrodes (Blue sensor; Medicotest Inc., Olstykke, Denmark) with an interelectrode distance of $20 \mathrm{~mm}$ (center-to-center) were placed according to the SENIAM recommendations (http://www.seniam.org): the semitendinosus (ST), 50\% on the line extending between the ischial tuberosity and medial epicondyle; the gluteus maximus (Gmax), 50\% on the line extending between the sacrum and greater trochanter; the lumbar multifidus (MF), at the level of the L5 spinous process on a line extending from the posterior superior iliac spine to the interspace between L1 and L2; the erector spinae (ES), at a 2-finger-width distance lateral from the spinous process of L1. A reference electrode was placed on the patella. EMG signals were recorded from those muscles on the leg extension side. Because preliminary analysis showed that the activities of the abdominal muscles (e.g., external oblique and transverse abdominis/internal oblique) were less than $5 \%$ of the maximal voluntary isometric contractions (MVICs) during prone hip extension in healthy individuals, these muscles were excluded from the analysis. Raw EMG signals, processed using bandpass filter with cut-off frequencies of $10 \mathrm{~Hz}$ and $500 \mathrm{~Hz}$ (CMR > 100dB), were amplified and collected with a sampling rate of $1,000 \mathrm{~Hz}$ using a 12-bit $\mathrm{A} / \mathrm{D}$ converter with a $\pm 5 \mathrm{~V}$ range (Telemyo 2400T V2; Noraxon USA Inc., Scottsdale, AZ). A trigger mechanism was used to synchronize the EMG measurements with the motion-capture system data.

The onset of muscle activity was determined using the cumulative sum (CUSUM) method (Ando et al., 2009; Brodin et al., 1993). First, EMG signals were rectified from $500 \mathrm{~ms}$ before the LED signal actuation to 1,000 $\mathrm{ms}$ after the signal; the background EMG signals were also averaged over the $500 \mathrm{~ms}$ period prior to the LED signal actuation. Then, the mean background EMG was subtracted from the rectified EMG. The rectified EMG was summed over 1,000 ms after the LED signal, and the resulting value was defined as $100 \%$. The EMG onset was defined as the point at which the cumulative sum of the rectified EMG reached a threshold of 5\% (Fig. 2). When the EMG onset was judged to be inappropriate, by visual inspection, the threshold was changed with a step of $1 \%$. The EMG onset time was determined by a blinded investigator.

The reaction time to the LED signal for the prime mover (the semitendinosus) was also evaluated. To investigate the temporal firing pattern among the hip and trunk muscles, the relative differences in the onset times between each muscle and the prime mover was calculated (Chance-Larsen et al., 2010; Lehman et al., 2004). A positive value indicated that the semitendinosus muscle was activated earlier.

A sub-maximal voluntary isometric contractions (sub-MVICs) was a more reliable measure than was maximal contraction as a method of normalization, particularly in patients unable to produce maximum exertions (Marras and Davis, 2001; Dankaerts et al., 2004). The prone hip extension from the $0^{\circ}$ hip flexion against gravity for $3 \mathrm{~s}$ was used as the sub-MVICs for the ST. The prone double leg raise with knees bent was used as the sub-MVICs for the Gmax, MF, and ES. These involved having 
the participant in a prone position with the knee bent to $90^{\circ}$ and both knees lifted off the ground for 3 s (Dankaerts et al., 2004).

For each individual muscle, the average root-mean-squares (RMSs) EMG amplitude was defined as a time window from the onset of each muscle activity to the termination of the movement of the knee marker. The average RMSs EMG amplitude of the each muscle was normalized to each of the sub-MVICs. In addition to the analysis of absolute muscle activity, we calculated muscle activity ratios among muscles in the lumbopelvic region. It is generally accepted that segment motion is affected by the relative muscle force of multiple muscles attached to the segment. From this point of view, analysis of not only absolute muscle activity but also the ratio of muscle activity around a segment has been emphasized, particularly in the scapular region (Cools et al., 2007; Ludewig et al., 2004). Although EMG is not a direct measure of muscle force production, comparative analysis between patients and healthy individuals would give relevant information about the alterations of relative contributions of each muscle to a movement. After normalization, Gmax/ST was calculated to index the balance of hip extensor muscle activity, MF/ES to index the balance of trunk muscle activity, $(\mathrm{MF} \times 2) /(\mathrm{Gmax}+\mathrm{ST}),(\mathrm{ES} \times 2) /(\mathrm{Gmax}+\mathrm{ST})$, and $(\mathrm{ES}+\mathrm{MF}) /(\mathrm{Gmax}+\mathrm{ST})$ to index the balance of hip and trunk muscle activity. Patients may not have decreased absolute muscle activity but rather decreased relative muscle activity of the lumbopelvic stabilizers.

\subsection{Hip extension ROM measurement}

20 We measured the passive range of motion (ROM) of hip extension, as restriction of the ROM may affect pelvic motion. Passive ROM of the hip extension was measured with participants in the supine position and their hip joints positioned at the edge of a table. The rater flexed the contralateral hip to assist in keeping the lumbar spine flat. The hip was then slowly extended by the rater toward the downward-tilted table with the hip maintained in neutral abduction-adduction, and rotation. The end position was defined as the point at which the motion of the evaluated leg stopped while flexion of the contralateral hip was maintained by the rater. In this position, the angle between the horizontal surface of the support surface and the femur was measured. These methods of measurements have been reported to yield reliable results in prior studies (Van Dillen et al., 2000; Pua et al., 2008). One physiotherapist performed the passive movement of the subjects' limb. A second physiotherapist positioned the goniometer and recorded the measurement. The examiner was blind to the amount of movement measured. The measurements showed acceptable intra-rater reliability with intraclass correlation coefficient $\left(\mathrm{ICC}_{1,1}\right)$ values of 0.82 in the present study.

\subsection{Statistical analysis}

The means of the 5 trials were used for analysis. All variables were assessed for normality using a Shapiro-Wilk statistic and graphical methods, prior to statistical analysis. The primary outcome was tested using the two-sided Student's t-test or Mann-Whitney test, as required, to detect any significant differences in the dependent variables between the control and patient groups. In the 
patient group, the relationship of each muscle activity and onset timing, and hip extension ROM with the magnitude of pelvic angle changes was tested with Pearson's product moment correlation coefficient or Spearman's rank correlation coefficient, according to the normality of variables. Statistical significance was determined as $\mathrm{P}<0.05$ for all statistical tests. The Statistical Package for Social Sciences 17.0 (SPSS Inc.) was used for the analyses.

\section{Results}

Passive hip extension ROM was significantly less in the patient group than in the control group (Table 1). No significant differences were noted in the reaction times, maximum leg motion velocity, and the onsets of pelvic motion between the groups (Table 1).

Although inter-subject variability was noted, almost all of the patients with THA extended their hip accompanied by anterior pelvic tilt, ipsilateral pelvic lift, and ventral pelvic rotation (Fig. 3). There was a significant difference in the pelvic rotation angles, as compared to controls $(p<0.01)$.

EMG activity of the ST was greater in the patient group than in the control group ( $p<0.05$, Table 1). Although there was no significant difference in the EMG amplitude in the trunk muscles between the groups, MF muscle activity relative to the hip extensor muscles $((\mathrm{MF} \times 2) /(\mathrm{Gmax}+\mathrm{ST}))$ was decreased in the patient group compared to the control group $(\mathrm{p}<0.05$, Table 1$)$.

Onset timings of the muscles, for both groups, are presented in Fig. 4. Despite the inter-subject variability, the overall temporal pattern of EMG onset of each muscle was similar between the groups. However, MF EMG onset was significantly delayed in the patient group compared to the control group ( $\mathrm{p}<0.05$, Fig. 4).

An association was seen between the EMG onset time and the EMG amplitude to increase pelvic motion within the patient group (Table 2). Delayed MF EMG onset, relative to that of the ST, was significantly related to the increase in the anterior pelvic tilt $(r=0.47, p<0.05$, Fig 5). Additionally, increased Gmax activity was significantly correlated to the increase in the ventral pelvic rotation $(\mathrm{r}=$ $-0.47, \mathrm{p}<0.05$, Fig. 5).

\section{Discussion}

In this study, patients with THA were compared to controls to determine the relationships between EMG amplitude, balance of muscle activity, and EMG onset timing and pelvic motion in the patient group. In the comparative analysis, patients with THA showed greater ventral pelvic rotation during prone hip extension than did healthy individuals. With regard to the EMG analysis, a decrease in the MF EMG amplitude relative to the hip extensors and a delayed MF activity onset, in addition to an increase in the ST EMG amplitude, were found in the patients with THA compared with 
controls. Furthermore, a correlation analysis within the patient group indicated that delayed onset of the MF was associated with increased anterior pelvic tilt during prone hip extension.

Although patients with THA showed smaller hip extension ROM than that of the controls, the passive angle of the hip extension in the patients ranged from $3^{\circ}$ to $20^{\circ}$. Therefore, the limited ROM

of the hip joint had little influence on the pelvic motion. In fact, there was no significant correlation between hip extension ROM and pelvic motion ( $r$ values: tilt 0.09 , oblique -0.18 , rotation -0.27 ) Since pelvic rotation was increased ventrally in the patients with THA, it is unlikely that increased pelvic rotation compensated for hip extension motion. Increased pelvic motion in the patients can be interpreted as an induced instability of the lumbopelvic region due to leg motion. Previous studies that examined gait in patients with THA demonstrated residual increased pelvic anterior tilt and rotation, and these increased pelvic motions were reported to be related to the limited hip flexion/extension motion (Miki et al., 2004; Perron et al., 2000). However, preoperative disturbances of pelvic motion were also described to be ameliorated early in the postoperative phase (Miki et al., 2004). In the present study, potential instability of the pelvis was observed, more than 6 months post-surgery, when internal perturbation was imposed on the lumbopelvic region by leg motion. The prone hip extension used in the present study might be a more sensitive task to expose lumbopelvic pathokinesiology than common gait analysis especially for patients with hip and lumbopelvic disabilities. ICC for motion analysis were within a range of 0.82 to 0.91 (ICC 1,1) and 0.96 to 0.98 (ICC 1,5). Thus, in the clinical setting, assessment of a typical motion pattern would be possible by observing several trials after a few practice trials, as done in the current study.

The muscle activity results indicated that patients with THA use an altered, and presumably inadequate, trunk muscle recruitment pattern to stabilize their lumbopelvic region. Patients with THA demonstrated delayed MF muscle onset timing relative to that of the ST, as compared with healthy subjects, despite the ST reaction times and speeds of leg motion not being significantly different between the groups. According to the criteria established by Hodges et al. (1996), onset of trunk muscle activity up to $50 \mathrm{~ms}$ after the onset of the prime mover is anticipatory muscle activation. The group mean MF onset time was $21.7 \mathrm{~ms}$ and $1.2 \mathrm{~ms}$ earlier than the onset of the ST in the control and patient groups, respectively. The latency of the MF can be interpreted within the feed-forward muscle activity. However, MF was activated later than the ST for $48 \%$ of the patients, whereas none of the healthy subjects activated their MF later than their ST. Therefore, temporally-coordinated activities of the hip and trunk muscles are altered in patients with THA.

Feedforward activation of the MF, accompanied with other abdominal muscles, is consistent with findings regarding rapid hip or arm movements while standing (Hodges and Richardson, 1997; Silfies et al., 2009). Despite delayed transverse abdominal muscle activity, MF activity is reportedly not delayed in patients with low back pain (Hodges and Richardson,1996), whereas delayed onset of MF activation was confirmed on the ipsilateral side of patients with sacroiliac joint pain, before the initiation of weight transfer (Hungerford et al., 2003). The delayed onset of MF activation was also observed in patients with low back pain during rapid arm raises, but only in patients with segmental 
instability (Silfies et al., 2009). Although still controversial, Silfies et al. (2009) suggests that delayed recruitment of the MF is linked to lumbopelvic instability rather than low back pain. The delayed onset observed in the patients with THA without lumbar spinal disease or symptoms may indicate that the altered timing of the MF activation does not necessarily yield clinical symptoms; however, it may increase the potential for instability in the lumbopelvic region. Patients with THA typically undergo joint replacement following an approximately 10-year duration of hip osteoarthritis (Sasaki et al., 2005); therefore, altered patterns of pelvic motion and muscle recruitment may develop prior to THA and remain afterwards.

It is noteworthy that, delayed MF onset was significantly associated with increased anterior pelvic tilt within the patient group. This finding is consistent with previous observations in healthy young individuals (Tateuchi et al., 2012), suggesting that anticipatory MF firing is a fundamental mechanism for dynamic control of anteroposterior pelvic tilt. The MF functions by stabilizing the lumbopelvic region through generation of compressive forces while producing torque to create extension of the lumbar spine (MacDonald et al., 2006). Inadequate lumbopelvic stability at the initiation of leg motion may be due to delayed activation of the MF (which has a stabilizing effect on the lumbopelvic region), resulting in increased anterior pelvic tilt.

A significant factor that causes excess, abnormal pelvic rotation could not be determined in this study. Although increased EMG amplitude of the Gmax was associated with the pelvic rotation, this activity might be a compensatory action contributing to incremental hip extension associated with increased ventral pelvic rotation. Trunk muscle activity, and the associated rise in intra-abdominal pressure, has been confirmed to increase lumbar spine stability (Cholewicki et al., 1999; Essendrop et al., 2002; Hodges et al., 2005); however, the contraction of individual abdominal muscles is not adequate to maintain spinal stability (Stokes et al., 2011). Thus, perhaps the simple correlation analysis used in this study prevented detection of a relationship between the increased pelvic rotation and muscle activity. Alternatively, excessive action by the other hip external rotators, not measured in this study, may rotate the pelvis ventrally during hip extension.

Patients with THA were shown to have a significant decrease in the MF to hip extensors amplitude ratio, compared to the controls. However, the altered balance of hip and trunk muscle activity in patients is attributed to tendency to display an increased ST amplitude, rather than decreased MF amplitude. This finding suggests that patients with THA have an impaired balance between the hip and trunk muscles (balance between mover and lumbopelvic stabilizer). Vogt and Banzer (1997) and Guimarães et al. (2010) investigated EMG onset timing, as well as individual muscle amplitudes, in the hip and trunk during their research on prone hip extension. However, other investigators, apart from Tateuchi et al. (2012) have not examined the activity of the MF and the balance between mover and stabilizer muscles during prone hip extension. The comprehensive assessment of spatiotemporal muscle recruitment patterns, in the current study, allowed the description of some of the underlying attributes of the coordination disorder among hip and trunk muscles for patients with THA. 
This study has several limitations. Although group differences were demonstrated in MF onset timings and the relative amplitudes of the MF to hip extensors, deep and superficial MF muscle activities were not measured individually because of the use of surface EMG. As reported in patients with low back pain (MacDonald et al., 2006), changes in activation patterns may be more explicit in deep fibers of the muscle (e.g., deep fibers of the MF) rather than in superficial fibers, even in patients with THA. In addition, detection of muscle activation delay in the clinical setting might be difficult without using EMG. An evaluation method that can be used in the clinical setting remains to be developed. Although prone hip extension is useful for evaluating the pathokinesiology of the lumbopelvic region, it is unknown whether these findings may be generalized to patients when standing. Additionally, only patients without hip pain were recruited and none of the patients complained of low back pain during the experimental task. Therefore, the results may not be valid in a population of patients with pain, as patients with hip or low back pain have been observed to show different neuromuscular controls and movement strategies (Dubois et al., 2011). However, the current study identified changes in kinematics and muscle recruitment patterns, without interference from joint pain. Impairment of motor control in the lumbopelvic region would represent deficiency of the stability of the base for hip movement. In that sense, it might be a critical issue for patients with THA, even in the absence of pain. The current study suggests that patients with THA who tend to tilt the pelvis anteriorly during leg motion require selective functional improvement of the lumbar multifidus.

In conclusion, patients with THA demonstrated pelvic instability and temporally altered MF muscle activity during prone hip extension. Importantly, the results of this study help to elucidate the relationship between changes in pelvic motion during leg motion and changes in muscle activity patterns of the trunk stabilizing muscles. Further research is necessary to determine whether motor training of the stabilizer muscles could prevent lumbopelvic instability in patients with THA.

\section{Acknowledgements}

We thank Kazuki Uemura for his assistance with data recording. Financial support was provided by the Grant-in-Aid for Young Scientists (B) from the Ministry of Education, Culture, Sports, 30 Science and Technology.

\section{References}

Ando S, Yamada Y, Tanaka T, Oda S, Kokubu M. Reaction time to peripheral visual stimuli during exercise under normoxia and hyperoxia. Eur J Appl Physiol 2009;106(1):61-9.

Beazell JR, Mullins M, Grindstaff TL. Lumbar instability: an evolving and challenging concept. J Man Manip Ther 2010;18(1):9-14. 
Ben-Galim P, Ben-Galim T, Rand N, Haim A, Hipp J, Dekel S, Floman Y. Hip-spine syndrome: the effect of total hip replacement surgery on low back pain in severe osteoarthritis of the hip. Spine 2007;32(19):2099-102.

Brodin P, Miles TS, Türker KS. Simple reaction-time responses to mechanical and electrical stimuli in human masseter muscle. Arch Oral Biol 1993;38(3):221-6.

Chance-Larsen K, Littlewood C, Garth A. Prone hip extension with lower abdominal hollowing improves the relative timing of gluteus maximus activation in relation to biceps femoris. Man Ther 2010;15(1):61-5.

Cholewicki J, Juluru K, McGill SM. Intra-abdominal pressure mechanism for stabilizing the lumbar spine. J Biomech 1999;32(1):13-7.

Cools AM, Declercq GA, Cambier DC, Mahieu NN, Witvrouw EE. Trapezius activity and intramuscular balance during isokinetic exercise in overhead athletes with impingement syndrome. Scand J Med Sci Sports 2007;17(1):25-33.

Dankaerts W, O'Sullivan PB, Burnett AF, Straker LM, Danneels LA. Reliability of EMG measurements for trunk muscles during maximal and sub-maximal voluntary isometric contractions in healthy controls and CLBP patients. J Electromyogr Kinesiol 2004;14(3):333-42.

Dubois JD, Piché M, Cantin V, Descarreaux M. Effect of experimental low back pain on neuromuscular control of the trunk in healthy volunteers and patients with chronic low back pain. J Electromyogr Kinesiol 2011;21(5):774-81.

Essendrop M, Andersen TB, Schibye B. Increase in spinal stability obtained at levels of intra-abdominal pressure and back muscle activity realistic to work situations. Appl Ergon 2002;33(5):471-6.

Guimarães CQ, Sakamoto AC, Laurentino GE, Teixeira-Salmela LF. Electromyographic activity during active prone hip extension did not discriminate individuals with and without low back pain. Rev Bras Fisioter 2010;14(4):351-7.

Hodges PW, Richardson CA. Inefficient muscular stabilization of the lumbar spine associated with low back pain. A motor control evaluation of transversus abdominis. Spine 1996;21(22):2640-50.

Hodges PW, Richardson CA. Contraction of the abdominal muscles associated with movement of the lower limb. Phys Ther 1997;77(2):132-144.

Hodges PW, Moseley GL, Gabrielsson A, Gandevia SC. Experimental muscle pain changes feedforward postural responses of the trunk muscles. Exp Brain Res 2003;151(2):262-71.

Hodges PW, Eriksson AE, Shirley D, Gandevia SC. Intra-abdominal pressure increases stiffness of the lumbar spine. J Biomech 2005;38(9):1873-80.

Hu H, Meijer OG, van Dieën JH, Hodges PW, Bruijn SM, Strijers RL, Nanayakkara PW, van Royen $\mathrm{BJ}, \mathrm{Wu} \mathrm{W}, \mathrm{Xia} \mathrm{C}$. Muscle activity during the active straight leg raise (ASLR), and the effects of a pelvic belt on the ASLR and on treadmill walking. J Biomech 2010;43(3):532-39.

Hungerford B, Gilleard W, Hodges P. Evidence of altered lumbopelvic muscle recruitment in the presence of sacroiliac joint pain. Spine 2003;28(14):1593-600. 
Janda V. Evaluation of muscular imbalance. In: Liebenson C, editor. Rehabilitation of the spine: a practitioner's manual. 1st ed. Baltimore: Lippincott Williams \& Wilkins, 1996: 97-112.

Lehman GJ, Lennon D, Tresidder B, Rayfield B, Poschar M. Muscle recruitment patterns during the prone leg extension. BMC Musculoskelet Disord 2004;5:3.

Ludewig PM, Hoff MS, Osowski EE, Meschke SA, Rundquist PJ. Relative balance of serratus anterior and upper trapezius muscle activity during push-up exercises. Am J Sports Med 2004;32(3):484-93.

MacDonald DA, Moseley GL, Hodges PW. The lumbar multifidus: does the evidence support clinical beliefs? Man Ther 2006;11(4):254-63.

Marras WS, Davis KG. A non-MVC EMG normalization technique for the trunk musculature. J Electromyogr Kinesiol 2001;11(1):1-9.

McNamara MJ, Barrett KG, Christie MJ, Spengler DM. Lumbar spinal stenosis and lower extremity arthroplasty. J Arthroplasty 1993; 8(3):273-7.

Miki H, Sugano N, Hagio K, Nishii T, Kawakami H, Kakimoto A, Nakamura N, Yoshikawa H. Recovery of walking speed and symmetrical movement of the pelvis and lower extremity joints after unilateral THA. J Biomech 2004;37(4):443-55.

Offierski CM, MacNab I. Hip-spine syndrome. Spine 1983; 8(3):316-21.

Panjabi MM. Clinical spinal instability and low back pain. J Electromyogr Kinesiol 2003;13:371-9.

Parvizi J, Pour AE, Hillibrand A, Goldberg G, Sharkey PF, Rothman RH. Back pain and total hip arthroplasty: a prospective natural history study. Clin Orthop Relat Res 2010;468(5):1325-30.

Perron M, Malouin F, Moffet H, McFadyen BJ. Three-dimensional gait analysis in women with a total hip arthroplasty. Clin Biomech 2000;15(7):504-15.

Prather H, Van Dillen LR, Kymes SM, Armbrecht MA, Stwalley D, Clohisy JC. Impact of coexistent lumbar spine disorders on clinical outcomes and physician charges associated with total hip arthroplasty. Spine J 2012. doi: 10.1016/j.spinee.2011.11.002.

Pua YH, Wrigley TV, Cowan SM, Bennell KL. Intrarater test-retest reliability of hip range of motion and hip muscle strength measurements in persons with hip osteoarthritis. Arch Phys Med Rehabil 2008;89(6):1146-54.

Sahrmann SA. Diagnosis and treatment of movement impairment syndromes. 1st ed. Missouri: Mosby, 2002.

Sasaki K, Senda M, Ishikura T, Ota H, Mori T, Tsukiyama H, Hamada M, Shiota N. The relationship between ambulatory ability before surgery and the D-dimaer value after total hip arthroplasty: The evaluation of ambulatory ability by the Timed "Up \& Go" test. Acta Med Okayama 2005;59(5):225-30.

Scholtes SA, Gombatto SP, Van Dillen LR. Differences in lumbopelvic motion between people with and people without low back pain during two lower limb movement tests. Clin Biomech 2009;24(1):7-12.

Silfies SP, Mehta R, Smith SS, Karduna AR. Differences in feedforward trunk muscle activity in 
subgroups of patients with mechanical low back pain. Arch Phys Med Rehabil 2009;90(7):1159-69.

Stokes IA, Gardner-Morse MG, Henry SM. Abdominal muscle activation increases lumbar spinal stability: analysis of contributions of different muscle groups. Clin Biomech 2011;26(8):797-803.

Stupar M, Côté P, French MR, Hawker GA. The association between low back pain and osteoarthritis of the hip and knee: A population based cohort study. J Manipulative Physiol Ther 2010;33(5):349-54.

Tateuchi H, Taniguchi M, Mori N, Ichihashi N. Balance of hip and trunk muscle activity is associated with increased anterior pelvic tilt during prone hip extension. $\mathrm{J}$ Electromyogr Kinesiol 2012;22(3):391-7.

Van Dillen LR, McDonnell MK, Fleming DA, Sahrmann SA. Effect of knee and hip position on hip extension range of motion in individuals with and without low back pain. J Orthop Sports Phys Ther 2000; 30(6): 307-16.

15 Vogt L, Banzer W. Dynamic testing of the motor stereotype in prone hip extension from neutral position. Clin Biomech 1997;12(2):122-27. 
Table 1

Descriptive data of hip and trunk muscle EMG activity and EMG ratio during prone hip extension

\begin{tabular}{|c|c|c|c|c|}
\hline & \multicolumn{2}{|c|}{ Controls } & \multicolumn{2}{|c|}{ Patients with THA } \\
\hline & Mean & SD & Mean & SD \\
\hline Passive hip extension ROM $\left(^{\circ}\right)$ & 16.5 & 4.3 & 11.2 & $3.8 * *$ \\
\hline Reaction time (ms) & 281.6 & 48.0 & 276.2 & 51.0 \\
\hline Maximum leg motion velocity (mm/sec.) & 488.0 & 180.8 & 469.2 & 153.0 \\
\hline Onset of pelvic motion (\%) & 29.2 & 23.0 & 20.1 & 21.1 \\
\hline \multicolumn{5}{|l|}{ Normalized RMSs EMG amplitude (\%) } \\
\hline Semitendinosus & 50.0 & 11.0 & 74.1 & $30.5 *$ \\
\hline Gluteus maximus & 30.7 & 29.3 & 43.2 & 32.1 \\
\hline Lumbar multifidus & 38.5 & 7.3 & 46.7 & 22.4 \\
\hline Erector spinae & 28.7 & 8.5 & 36.1 & 15.7 \\
\hline \multicolumn{5}{|l|}{ Ratio of the EMG } \\
\hline Gmax/ST & 0.58 & 0.42 & 0.58 & 0.29 \\
\hline MF/ES & 1.44 & 0.38 & 1.33 & 0.32 \\
\hline$(\mathrm{MF} \times 2) /(\mathrm{Gmax}+\mathrm{ST})$ & 1.10 & 0.43 & 0.85 & $0.26 *$ \\
\hline$(\mathrm{ES} \times 2) /(\mathrm{Gmax}+\mathrm{ST})$ & 0.81 & 0.38 & 0.65 & 0.16 \\
\hline$(\mathrm{MF}+\mathrm{ES}) /(\mathrm{Gmax}+\mathrm{ST})$ & 0.95 & 0.39 & 0.75 & 0.20 \\
\hline
\end{tabular}

(Footnote for Table 1)

Gmax $=$ Gluteus maximus, $\mathrm{ST}=$ Semitendinosus, $\mathrm{MF}=$ Lumbar multifidus, and ES = Erector spinae .

$* \mathrm{p}<0.05, * * \mathrm{p}<0.01$ 
Table 2

$R$ values for correlations between pelvic angle changes and EMG onset timing or EMG amplitude and EMG ratio

\begin{tabular}{lccc}
\hline & $\begin{array}{c}\text { Pelvic tilt } \\
(+: \text { anterior tilt })\end{array}$ & $\begin{array}{c}\text { Pelvic oblique } \\
(+: \text { ipsilateral rise })\end{array}$ & $\begin{array}{c}\text { Pelvic rotation } \\
(+: \text { dorsal rotation })\end{array}$ \\
\hline EMG onset timing $^{\mathrm{a}}$ & & & \\
$\quad$ Gluteus maximus & 0.27 & -0.05 & 0.39 \\
Lumbar multifidus & $0.47^{*}$ & 0.16 & 0.01 \\
Erector spinae & 0.36 & 0.17 & 0.14 \\
EMG amplitude and EMG ratio & & & \\
Semitendinosus & -0.21 & 0.01 & -0.42 \\
Gluteus maximus & -0.10 & -0.09 & $-0.47^{*}$ \\
Lumbar multifidus & -0.34 & 0.30 & -0.31 \\
Erector spinae & -0.28 & 0.13 & -0.38 \\
Gmax/ST & 0.10 & 0.03 & -0.13 \\
MF/ES & -0.08 & 0.35 & 0.18 \\
$(\mathrm{MF} \times 2) /(\mathrm{Gmax}+\mathrm{ST})$ & -0.26 & 0.41 & 0.25 \\
$(\mathrm{ES} \times 2) /(\mathrm{Gmax}+\mathrm{ST})$ & -0.25 & 0.24 & 0.22 \\
$(\mathrm{MF}+\mathrm{ES}) /(\mathrm{Gmax}+\mathrm{ST})$ & -0.27 & 0.37 & 0.25 \\
\hline
\end{tabular}

(Footnote for Table 2)

${ }^{\text {a }}$ EMG onset time of the each muscle relative to that of the semitendinosus.

Gmax = Gluteus maximus, $\mathrm{ST}=$ Semitendinosus, $\mathrm{MF}=$ Lumbar multifidus, and ES = Erector spinae.

$* \mathrm{p}<0.05$ 




a.

b.

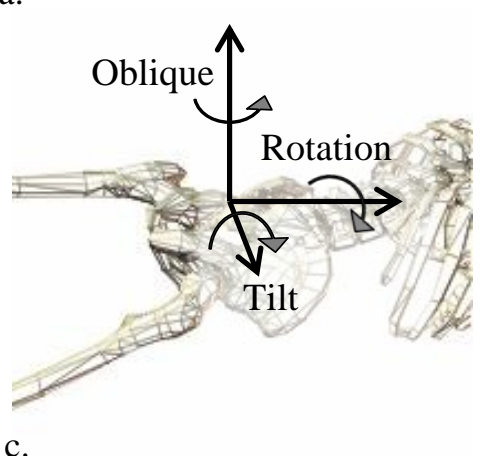

c.

Fig. 1 


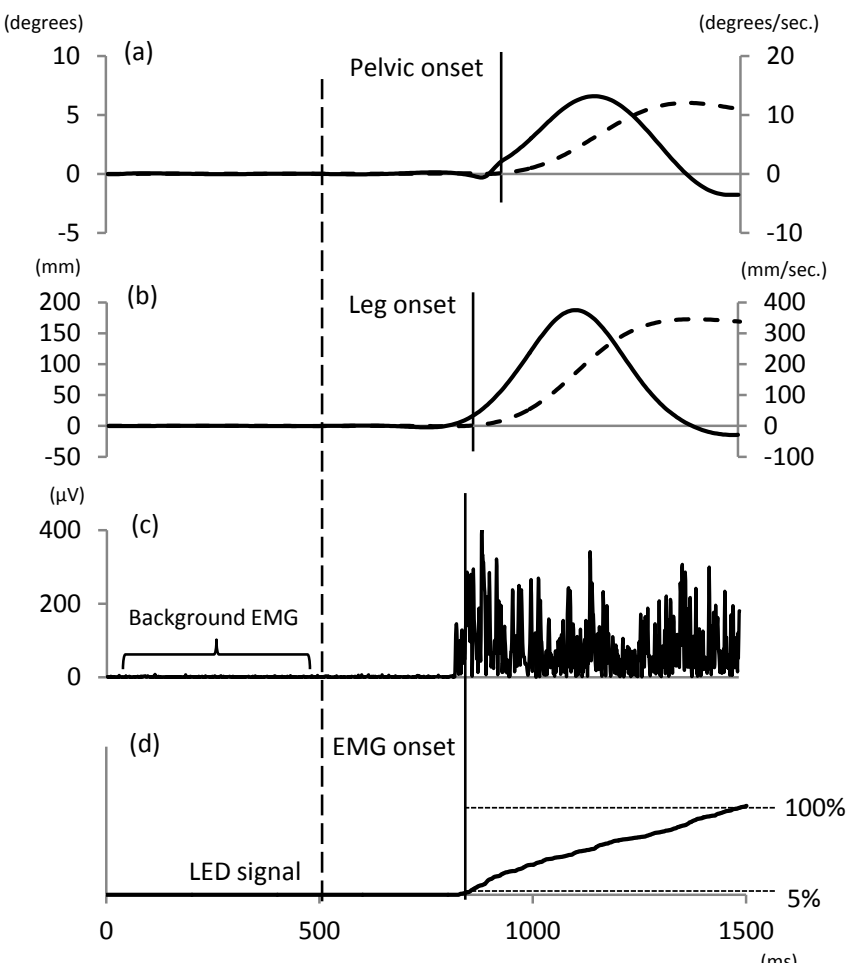

Fig. 2 


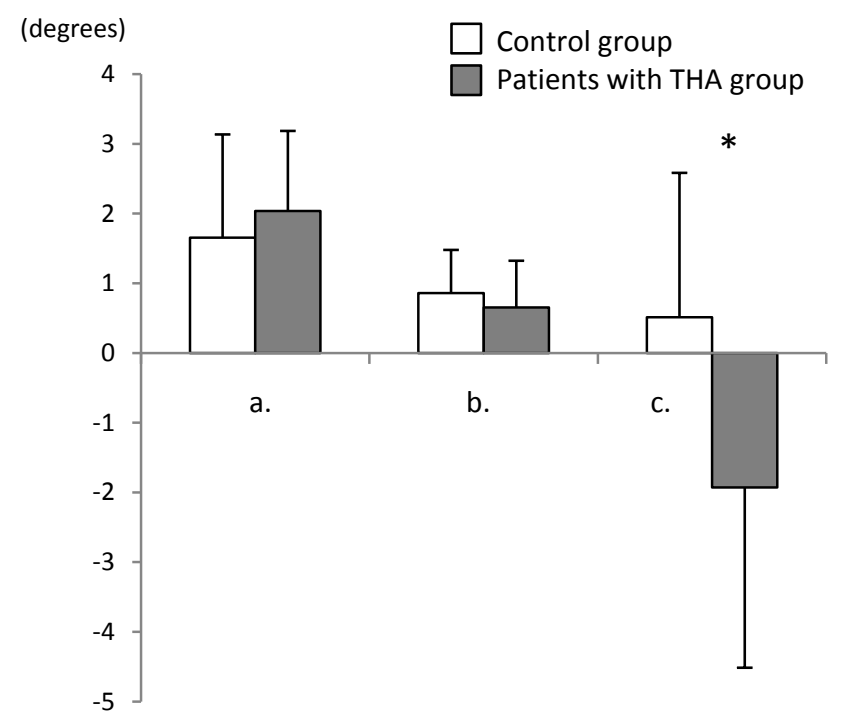

Fig. 3 


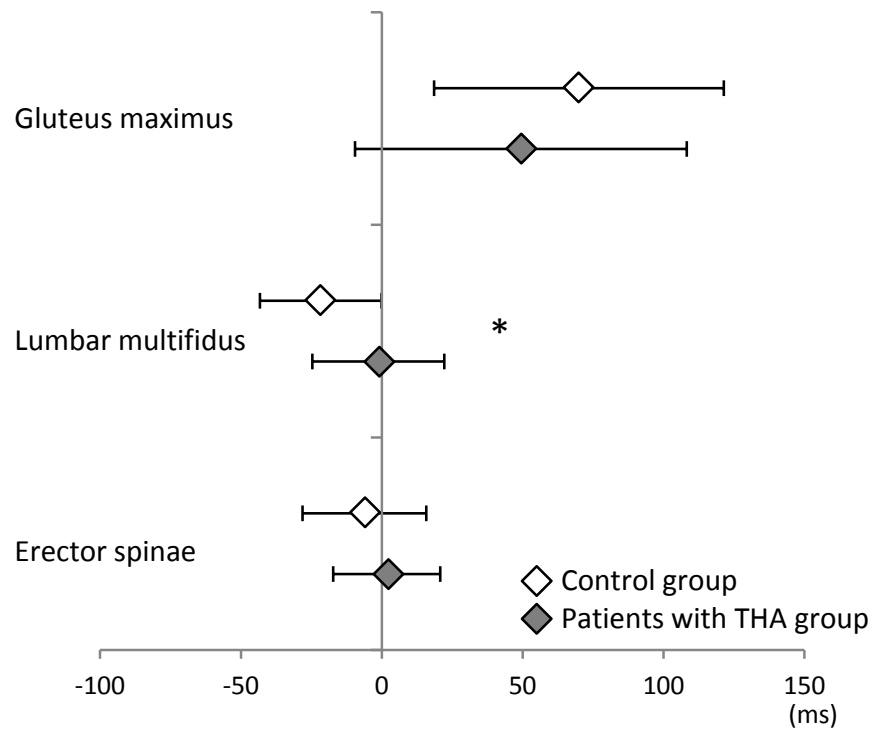

Fig. 4 

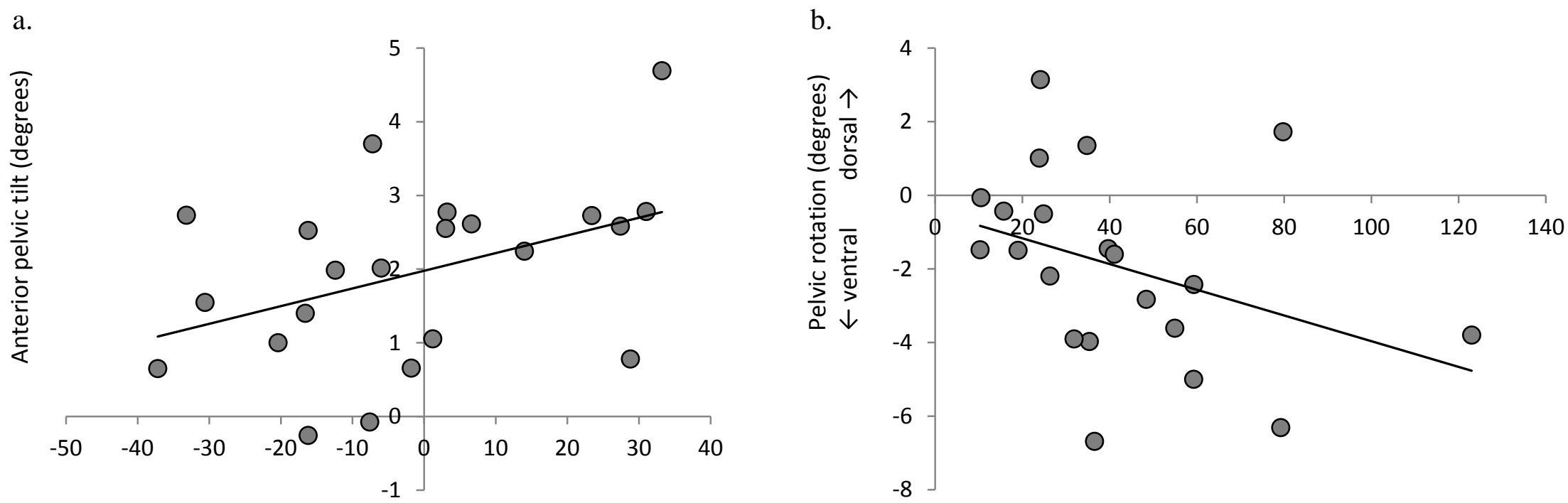

Lumbar multifidus EMG onset time relative to the semitendinosus onset (ms)

Normalized EMG amplitude of the gluteus maximus (\%)

Fig. 5 
Fig. 1. Active prone hip extension from the $30^{\circ}$ (a) to the $0^{\circ}$ hip-flexed position (b). Each participant was instructed to move as rapidly as possible in response to the LED signal. Markers were attached on the bilateral posterior superior iliac spine, top of the iliac crest, unilateral greater trochanter, lateral epicondyle of the femur, and lateral malleolus. Tilt, oblique, and rotation of the pelvis were calculated (c).

Fig.2. Kinematics and EMG data from a representative control participant during a prone hip extension. Following traces are shown: angle of the anterior pelvic tilt (a; dashed line) and velocity of the pelvic tilt (a; solid line), vertical motion of the lateral epicondyle ( $b$; dashed line) and velocity of the lateral epicondyle ( $b$; solid line), rectified EMG of the semitendinosus (c), and cumulative sum of the rectified EMG of the semitendinosus to which mean background EMG was subtracted (d). The vertical dashed line denotes LED signal, the solid line denotes onset of the anterior pelvic tilt, leg motion, and muscle activity.

Fig.3. The mean and SD angle changes of the pelvis tilt (a), oblique (b), and rotation (c) during prone hip extension. Positive values indicate anterior tilt (a), ipsilateral rise (b), and dorsal rotation (c). Asterisk indicates significant difference between groups $(\mathrm{p}<0.05)$.

Fig.4. The mean and SD EMG onset time of the gluteus maximus, lumbar multifidus, and erector spinae relative to that of the semitendinosus (time 0 ). Positive onset times indicate a later onset relative to ST. Asterisk indicates significant difference between groups $(\mathrm{p}<0.05)$.

Fig. 5. Delayed lumbar multifidus EMG onset relative to that of the semitendinosus was significantly correlated to increased anterior pelvic tilt during prone hip extension in the patient group $(a ; r=0.47, p<0.05)$. Positive onset times indicate a later onset relative to ST (a). Increase of the gluteus maximus EMG was significantly related to increase of the ventral pelvic rotation $(b ; \mathrm{r}=-0.47, \mathrm{p}<0.05)$. 